\title{
Apropiación de terminología médica por estudiantes de primer curso del grado en medicina a través de prensa diaria
}

\author{
Begoña Cantabrana, Brezo Diez, Javier Bordallo, Manuel Sánchez, Agustín Hidalgo
}

Objetivo. Presentar y analizar una tarea formativa de apropiación de terminología médica y científica por los estudiantes de primer curso de medicina.

Sujetos y métodos. Participaron los 161 alumnos matriculados en primer curso que fueron distribuidos en grupos de cinco o seis para realizar el trabajo propuesto en forma de seminario. Los alumnos seleccionaron noticias relacionadas con ciencia y medicina publicadas en diarios de ámbito nacional o regional para estudiar diferentes características. Las instrucciones para la realización de la tarea, así como algunos ejemplos, estuvieron disponibles en el campus virtual de la universidad al comienzo de curso.

Resultados. Los alumnos seleccionaron 103 artículos, de los que sólo seis trataban de ciencias naturales no relacionadas con la medicina, predominando en estos últimos los dedicados a neurología. En esos 103 artículos se identificaron 316 términos médicos; otros 219 términos fueron seleccionados en artículos generales. El 88,3\% de los términos fueron documentados mediante distintos diccionarios. El 51,5\% de los alumnos contestaron una encuesta sobre la actividad formativa en la que otorgaron una calificación de 3,2 puntos sobre 5 .

Conclusiones. El estudio demuestra que las noticias sobre medicina y salud son habituales en la prensa nacional y regional. Los alumnos consideraron que la actividad favorece la adquisición de vocabulario médico y científico, a la vez que facilita la aproximación a los aspectos sociales de la medicina.

Palabras clave. Aprendizaje activo. Autoaprendizaje. Divulgación científica. Noticias de prensa. Terminología médica.

Use of newspaper for the terminology appropriation by first year medical students

Farmacología. Departamento de Medicina. Universidad de Oviedo. Instituto Universitario de Oncología del Principado de Asturias (IUOPA). Oviedo, Asturias, España.

Correspondencia:

Dra. Begoña Cantabrana. Farmacología. Departamento de Medicina. Facultad de Medicina y Ciencias de la Salud. Universidad de Oviedo. Julián Clavería, 6. E-33006 Oviedo (Asturias).

Fax: +34985103551.

E-mail: bego@uniovi.es

Conflicto de intereses: No declarado.

Conflict of interests: None declared.

(c) 2013 FEM

Aim. To describe and analyze a formative task of appropriation of medical and scientific terminology by students of first year at medical school.

Subjects and methods. Participants were 161 students distributed in groups of five or six to perform the proposed assignments in form of seminars. The students selected science and medicine news, published in national and regional newspapers, to analyze different characteristics of these articles. The instructions for the task as well as some examples were available in the university's virtual campus at the beginning of the course.

Results. Students chose 103 news; only six of natural sciences and the rest related to Medicine, being predominant those related to neurology. Medical terms identified in those articles were 316. Moreover 219 items in general news were chosen. The $88.3 \%$ of the terms were documented via consulting dictionaries. The $51.5 \%$ of the students answered a survey in which, the task got an average score of 3.2 out of 5 possible points.

Conclusions. The study shows that medicine and health information is common in national and regional press. The students think that this activity promotes medical and scientific vocabulary acquisition while it favors the approach to the social aspects of medicine.

Key words. Active learning. Medical terminology. Press news. Scientific dissemination. Self-learning.

\section{Introducción}

Todo campo específico del saber necesita un vocabulario y una retórica especiales para poder expresar correctamente y con la mayor precisión posible las ideas y los conceptos propios de esa parcela del conocimiento [1]. La medicina no es una excepción. El lenguaje en medicina es importante para la intercomunicación gremial entre los colegas, la información al paciente, la información a los familiares 
del paciente y por el valor de la palabra como terapia [2]. Así mismo, el lenguaje médico publicado representa el máximo escalón de comunicación, sobre todo en los medios de comunicación de masas, donde se expone a toda la sociedad, convirtiéndose en un lenguaje externo al que puede acceder todo el público.

La importancia del lenguaje en el aprendizaje de una disciplina es innegable porque es imposible aprender una ciencia sin conocer el lenguaje en el que esa ciencia se expresa y sin saber interpretar correctamente su discurso. En el caso de la medicina, se estima que los alumnos deben aprender unas 15.000 palabras en los tres primeros cursos de sus estudios [3]. De la misma forma se considera que un mal aprendizaje de la terminología especializada constituye uno de los factores más importantes del fracaso académico de los alumnos.

Por otra parte, la terminología médica es un instrumento básico de comunicación de la biomedicina científica moderna que circula y se difunde por los medios de comunicación social, el discurso político, la literatura y los textos científicos y humanísticos, hasta llegar incluso al lenguaje coloquial. En la creación y difusión del léxico médico intervienen no sólo factores científicos, sino también extracientíficos, y mucho más cuando los términos se incorporan a la lengua general y pasan a formar parte del léxico común. Así mismo, los términos científicos y médicos adquieren, en la lengua general, una carga cultural añadida que los convierte en instrumentos idóneos para expresar contenidos ideológicos, explotando sus sentidos connotativos $[4,5]$.

Bourdieu [6] ha llamado la atención sobre el hecho de que la dificultad de transmisión de los productos de investigación guarda relación con el lenguaje. De ahí la necesidad de que los alumnos aprendan a identificar en los medios de comunicación de masas términos relacionados con su actividad futura y lo hagan en vehículos de expresión accesibles a todo el público y aprendan el significado que se les atribuye, el contexto en que se usan y las figuras literarias en que se implican. Se estima que 'un diccionario de medicina puede contener 80.000 términos, de los que específicos de la medicina pueden ser unos 55.000' [7]. Por esta magnitud, se entiende que la terminología médica es un verdadero lenguaje que debe aprender el estudiante de forma aditiva a la complejidad que ofrece la ciencia médica. Una dificultad añadida es que, en los estudios preuniversitarios, no ha recibido una preparación adecuada para este aprendizaje y en la universidad sólo recibe instrucciones, con suerte, en la asignatura sobre historia de la medicina.
Por nuestra parte, consideramos que el aprendizaje del vocabulario médico es básico para la adquisición de conceptos y conocimientos médicos. Por eso hemos recurrido, dentro de la asignatura 'Introducción a la medicina, documentación y método científico, al ejercicio que describimos en este trabajo para iniciar a los alumnos del primer curso del grado en medicina en la importancia de identificar palabras médicas y científicas, que irán incorporando a su vocabulario para conformar un verdadero léxico profesional y un vehículo de comunicación en ciencia médica [8].

\section{Sujetos y métodos \\ Organización y seguimiento de la actividad}

La asignatura en la que se desarrolla el ejercicio 'Introducción a la medicina, documentación y método científico' tiene seis créditos ECTS, con 60 horas presenciales (58 horas de actividades presenciales y dos de examen escrito) y 90 horas no presenciales, que se imparte en el primer semestre del primer curso del grado en medicina. Las actividades presenciales se distribuyeron en 28 clases teóricas (incluyendo dos conferencias invitadas), seis horas de prácticas, 20 de seminarios y dos de tutoría grupal. La actividad descrita en este trabajo se incluyó dentro de los seminarios.

\section{Grupos de alumnos y programación de la tarea}

En el curso se matricularon 161 alumnos, de los que 152 eran de nueva matrícula y nueve adaptados de la licenciatura en medicina. De ellos, 139 accedieron desde la prueba de acceso a la universidad, ocho por titulados universitarios, dos por mayores de 25 años, otros dos accedieron por convalidación parcial de estudios extranjeros y uno por traslado desde otra universidad. Los 161 alumnos matriculados se distribuyeron en 32 grupos de trabajo, compuestos por cinco o seis alumnos, que se mantuvieron estables a lo largo del semestre, de la misma forma que describimos en un trabajo previo [9]. Los seminarios se realizaron con ocho grupos de trabajo (40 alumnos). En cada sesión, un alumno de cada uno de los grupos participantes debió exponer el trabajo realizado de forma conjunta y participativa. Al final de la actividad, el alumno ponente debía incorporar la presentación al portafolio del grupo. Esta información y las notas personales del profesor en el curso del seminario (exposición y debate) han sido los documentos utilizados para la realización del presente trabajo. 
Al comienzo del curso, cada grupo de trabajo tenía asignadas las tareas que debía realizar a lo largo del semestre para que pudieran organizar su tiempo adecuadamente. En el caso del seminario que comentamos, los alumnos tenían disponibles en el campus virtual de la universidad las instrucciones para su realización. Estas instrucciones contenían un objetivo (tomar consciencia de la dimensión social de la ciencia y la medicina a través de su presencia en los medios de comunicación) y una pormenorización de las tareas:

- Identificar noticias relacionadas con ciencia o medicina, aparecidas en un diario nacional o regional, durante un período de al menos siete días, y extraer la siguiente información: identificar la sección en que aparece, el tema que trata, los términos científicos y médicos utilizados y los conceptos que transmiten, establecer el tipo de noticia (divulgación, agencias, artículo invitado, reportaje de investigación...), atribuir importancia potencial a la noticia en el ámbito científico, político, social, económico, etc., y tipificar el presumible objetivo de la noticia.

- Identificar términos propios de la medicina (no necesariamente exclusivos de ella) en artículos generales (entendiendo por tales que su objetivo no es comunicar ciencia o medicina). En este caso se trata de identificar palabras que, en sentido metafórico o no, han salido del ámbito especializado y se han incorporado al lenguaje común.

De forma orientativa se les incluía una selección de tres noticias tomadas de la edición nacional del diario El País: la nota de agencia 'Científicos coreanos logran la primera clonación de embriones humanos con fines terapéuticos' y los artículos firmados por Malen Ruiz de Elvira 'Si te dimos una patada fue entre amigos. Cartas inéditas entre los científicos del ADN desde 1950 reflejan su rivalidad', del 30 de septiembre de 2010, y por Yolanda Monge 'Estados Unidos pide perdón a Guatemala por experimentar con pacientes y presos. Unas 700 personas fueron infectadas con sífilis y gonorrea en los años cuarenta', del 2 de octubre de 2010. Hemos elegido periódicos españoles porque diferentes autores han coincidido en considerar que la información que aportan, referida a la salud, es de buena calidad [10-13]. Así mismo, se les facilitó el acceso a algunas fuentes de referencia [14-16].

\section{Encuesta}

Con el fin de conocer la opinión de los alumnos sobre aspectos relacionados con el aprendizaje, los mé-
Figura 1. Distribución por temas de los artículos de contenido médico $(n=97)$ seleccionados por los alumnos. SNS: Sistema Nacional de Salud.

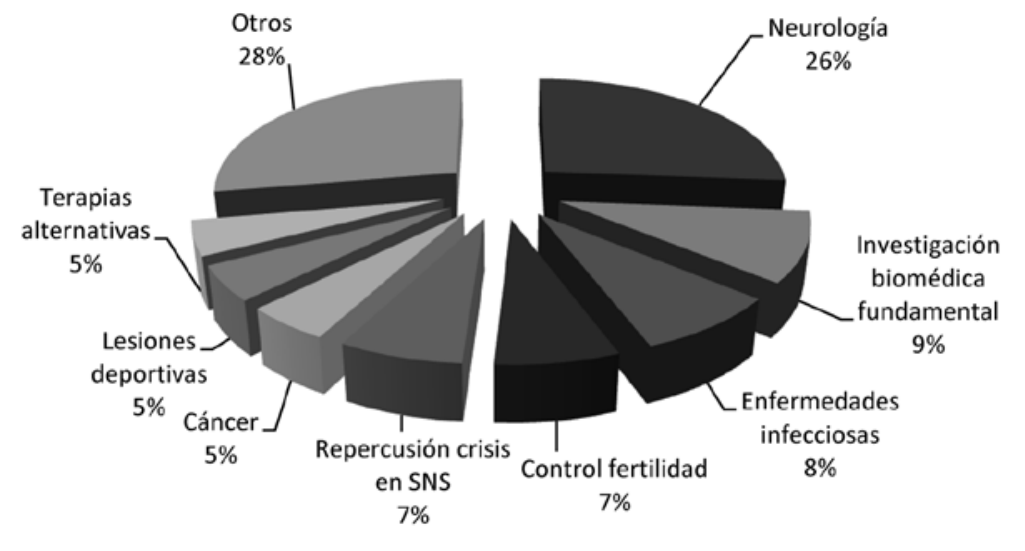

todos, el trabajo en grupo y la adquisición de competencias, diseñamos una encuesta de satisfacción en la que se incluían preguntas concretas sobre este seminario. Los alumnos cumplimentaron la encuesta, de forma voluntaria y anónima, a través del campus virtual, después de la realización del examen escrito $\mathrm{y}$ antes de hacer públicas las calificaciones.

\section{Resultados}

Los 32 grupos de alumnos presentaron en el seminario una selección de 103 artículos de contenido médico o científico. De ellos, sólo 6 (5,8\%) no estaban relacionados con aspectos de la medicina. El contenido médico estaba presente en el 94,2\%, con la distribución por temas que muestra la figura 1, en la que sobresalen como temas más seleccionados los relacionados con neurología. Las fuentes de documentación más consultadas fueron periódicos de tirada nacional, seguidos de los de ámbito regional, siendo El País (28,2\%) el más citado, y en segundo lugar, el diario regional La Nueva España (26,2\%) (Fig. 2).

Los alumnos clasificaron los artículos por su modalidad y consideraron que el 80,6\% tenían carácter divulgativo, repartiéndose el porcentaje restante entre ocho modalidades diferentes (Tabla I). Así mismo, consideraron que los objetivos más frecuentemente perseguidos por las noticias seleccionadas eran: comunicar datos de estudios clínicos $(17,5 \%)$, la relación beneficio-riesgo de medicamentos $(17,5 \%)$, la promoción de la salud $(14,6 \%)$ y dar 
Figura 2. Fuentes de las noticias seleccionadas.

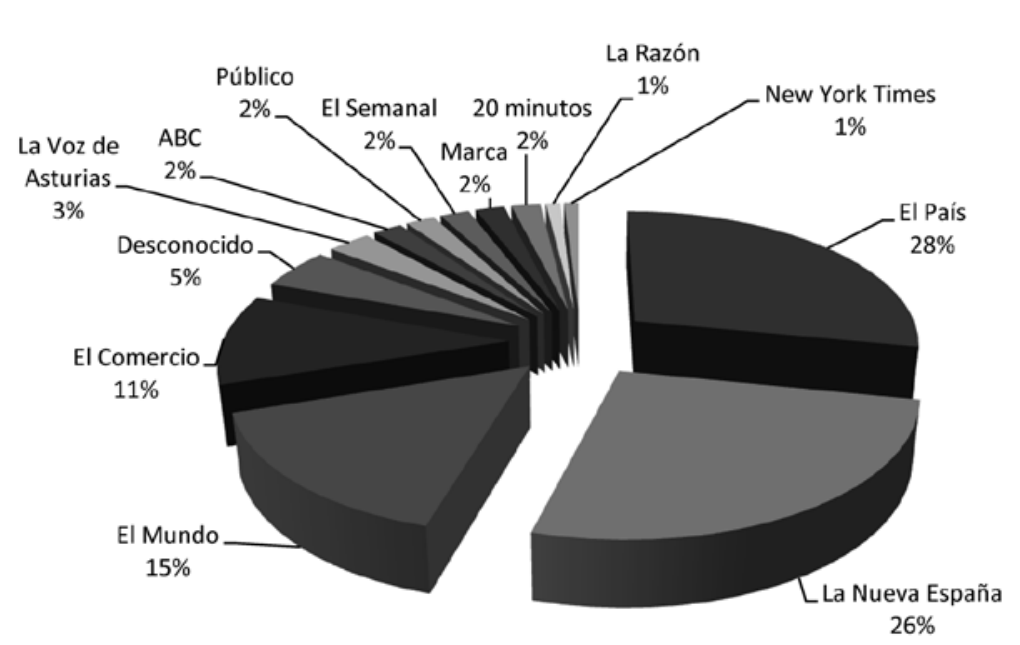

$26 \%$
Tabla I. Modalidad del artículo seleccionado $(n=103)$.

\begin{tabular}{lcc}
\hline & $n$ & $\%$ \\
\hline Divulgación & 83 & 80,6 \\
\hline Reportaje & 7 & 6,8 \\
\hline Entrevista & 4 & 3,8 \\
\hline Opinión & 3 & 2,9 \\
\hline Actualidad deportiva & 2 & 1,9 \\
\hline Ensayo & 1 & 1,0 \\
\hline Historia & 1 & 1,0 \\
\hline Actualidad económica & 1 & 1,0 \\
\hline Promocional & 1 & 1,0 \\
\hline
\end{tabular}

con términos propios de otra. Una breve selección de estas expresiones se muestra en la tabla IV.

Los estudiantes realizaron un ejercicio de aprendizaje de terminología porque el $88,3 \%$ de las palabras médicas o científicas identificadas en los textos fueron documentadas adecuadamente mediante el diccionario de la Real Academia Española, el de María Moliner y diferentes diccionarios online, como diccionario.net, diccionarios.com, wordreference.com, y algunas páginas de Internet de sociedades científicas o médicas, como fisterra.com, o la tradicional enciclopedia Espasa. En cambio, en un porcentaje elevado $(62,1 \%)$ de los trabajos presentados no fue posible identificar conclusiones personales de los alumnos.

La encuesta de satisfacción fue contestada por 83 alumnos, el 51,5\% de los matriculados. La asignatura ha recibido una calificación de satisfacción de 3,9 sobre 5 puntos, alcanzando el seminario que se describe en este estudio una puntuación de 3,2 puntos (inferior al 4,0 con que fue valorado globalmente el apartado de seminarios); se le reconoce la virtud de favorecer la adquisición de vocabulario médico y científico $(4,1$ frente a 5,0$)$ y que constituye una aproximación a los aspectos sociales de la medicina (4,2 frente a 5,0$)$. que de los 535 términos identificados entre ambos tipos de artículos, sólo $6(1,9 \%)$ no fueron términos médicos. Sólo el 20,4\% de las palabras se utilizaron en el texto en su sentido denotativo literal, mientras que el 79,6\% lo fue en sentido metafórico y formando parte de frases o expresiones que se ajustan al concepto de uso metafórico porque definen una cosa

\section{Discusión}

El número de los alumnos que cumplimentaron la encuesta es representativo de la opinión general del 
curso. La puntuación otorgada a la asignatura y a la actividad puede considerarse satisfactoria, aunque puede mejorarse. En todo caso, podemos considerar que se ha alcanzado el objetivo del seminario y sus competencias asociadas, ya que los estudiantes consideran que la actividad favorece la adquisición de vocabulario médico y científico, sobre todo al verse reforzado por la necesidad de documentar los términos identificados como exigen las instrucciones. Además, puede cumplir otra importante función, como es la aproximación a los aspectos sociales de la ciencia y la medicina, particularmente relevante en una población estudiantil de poco más de 18 años que trabajará en medio del dolor ajeno. Consideramos, además, que la actividad puede contribuir a la formación humanística del alumno e incentiva la comunicación científica (exponen los trabajos y, en ocasiones, realizan descripciones y ensayos) desde los parámetros del nuevo humanismo encarnado por científicos divulgadores y humanistas [17], perfil al que desde siempre han pertenecido los médicos y cuya contribución a la divulgación es demandada constantemente [18].

La selección de artículos realizada por los alumnos apunta dos aspectos orientativos:

- Sólo una pequeña proporción de artículos se refieren a ciencias naturales porque aquellos que tienen una base biológica están orientados a la biomedicina y la salud. El artículo dominante en este campo es el referido a los neutrinos, publicado con titulares de excepción porque podrían superar la velocidad de la luz y poner en evidencia la teoría de la relatividad de Einstein. Otros dos temas frecuentes han sido la celebración de un congreso de oftalmología auspiciado por un instituto médico local y, sobre todo, la enfermedad de Alzheimer, habida cuenta de la proliferación de artículos de prensa a propósito del Día Mundial del Alzheimer, celebrado el 21 de septiembre de 2011. Por tanto, hechos incidentales, como ya han demostrado los sucesivos trabajos de la Fundación Vila Casas recogidos en los Informes Quiral [16], condicionan la frecuencia de noticias en campos particulares. Llama la atención que un tema tan mediático como el cáncer ocupe un lugar poco relevante en la atracción de los alumnos que, como queda dicho, han demostrado tener poca inclinación por las ciencias naturales si no están orientadas a la medicina.

- Una muestra tentativa de las preferencias de los alumnos por el ejercicio de la profesión médica es que han elegido más artículos sobre medicina clínica, las consecuencias de los actos médicos y sobre educación para la salud que sobre la inves-
Tabla II. Objetivo de los artículos $(n=103)$.

\begin{tabular}{lll}
\hline & $n$ & $\%$ \\
\hline Comunicar datos de estudios clínicos & 18 & 17,5 \\
\hline Relación riesgo-beneficio de medicamentos y práctica clínica & 18 & 17,5 \\
\hline Promoción de la salud & 15 & 14,6 \\
\hline Comunicar datos de investigación biomédica fundamental & 14 & 13,6 \\
\hline Comunicar datos de ciencias naturales no biológicas & 5 & 4,8 \\
\hline Sensibilizar a la sociedad sobre enfermedades & 7 & 6,9 \\
\hline Funcionamiento del Sistema Nacional de Salud & 5 & 4,8 \\
\hline Promocionar productos (publicidad bajo diferentes soportes) & 5 & 4,8 \\
\hline Comunicar las repercusiones de la crisis económica en la salud & 6 & 5,8 \\
\hline Informar de lesiones deportivas & 4 & 3,9 \\
\hline Varios (entrevistas, historia de enfermedades o logros científicos) & 6 & 5,8 \\
\hline
\end{tabular}

Tabla III. Distribución por temas de los términos identificados en artículos generales y médicos.

\begin{tabular}{|c|c|c|c|c|}
\hline & \multicolumn{2}{|c|}{ Generales $(n=219)$} & \multicolumn{2}{|c|}{ Medicina $(n=316)$} \\
\hline & $n$ & $\%$ & $n$ & $\%$ \\
\hline Enfermedad/patología & 47 & 21,4 & 62 & 19,6 \\
\hline Estructuras anatómicas y elementos subcelulares a & 36 & 16,4 & 49 & 15,5 \\
\hline Función y estado del paciente & 30 & 13,7 & 36 & 11,4 \\
\hline Aspectos generales ${ }^{b}$ & 25 & 11,4 & 31 & 9,9 \\
\hline Sistema Nacional de Salud & 19 & 8,7 & 29 & 9,2 \\
\hline Medicamentos & 18 & 8,3 & 47 & 14,8 \\
\hline Especialidades médicas & 14 & 6,4 & 12 & 3,8 \\
\hline Acto médico & 13 & 5,9 & 14 & 4,4 \\
\hline Instrumentos y técnicas & 12 & 5,5 & 22 & 6,9 \\
\hline Terapias no farmacológicas & 5 & 2,3 & 6 & 1,9 \\
\hline Términos científicos no médicos & - & - & 6 & 1,9 \\
\hline
\end{tabular}

a Incluye términos de anatomía, histología, biología celular, etc.; b Tales como vida, muerte, médico, hombre, mujer, etc.

tigación biomédica fundamental. Posiblemente este hecho orienta sobre el carácter vocacional de la medicina y que la idea profesional de medi- 
Tabla IV. Ejemplos de expresiones metafóricas detectadas.

Sociedad alzheimica

El cáncer terrorista no deja de metastatizarse

Pese a tratarse de un consumo que está relacionado con la aparición de hasta 60 enfermedades, el alcohol goza casi de inmunidad social

Rajoy promete sacar a España de la crisis

Desde hace año y medio, los políticos sentían una especie de catarata personal que impedía que pudieran verse el uno al otro

Hay mucho político miope incapaz de ver más allá

El electrocardiograma [de Grecia] se parece al de un hombre muerto

Siete leyes sociales en estado terminal

El Estado sufre una enfermedad que no se está tratando de la forma adecuada y cuyo virus no hace más que mutar

cina se asocia al contacto con la clínica (con el cuidado del paciente) y no con la investigación fundamental.

Por otra parte, el hecho de que los alumnos otorguen relevancia a los contenidos de los artículos seleccionados sugiere que el mensaje transmitido en la prensa es potencialmente educativo (p. ej., los relacionados con promoción de la salud ocupan el tercer lugar en la selección) y, por tanto, útil a la hora de transmitir información a la ciudadanía, así como fuente de formación de una conciencia sanitaria, lo que confirma el trabajo de Cámara-Hurtado y López-Cerezo [15], quienes detectan una alta demanda de información en salud y una baja conformidad con la cantidad que se ofrece.

La fuente de los textos seleccionados es coherente porque periódicos de ámbito nacional ocupan la primera y tercera posición, y uno de ámbito regional, que se encuentra entre los diez periódicos españoles de mayor tirada, ocupa el segundo. Esto último puede explicar que noticias tan locales como un congreso de una especialidad médica celebrado en la localidad donde tiene su sede la universidad o artículos o entrevistas a médicos locales estén representadas en la selección. Todos los diarios dedican espacio a las noticias en secciones relevantes como los suplementos de ciencia o de salud en los periódicos nacionales y en el apartado de sociedad en los regionales y nacionales.

Los términos identificados se han documentado, generalmente, mediante el diccionario de la Real Aca- demia Española y diccionarios electrónicos, resaltando la ausencia de diccionarios médicos tradicionales en papel. Esto es coherente con la versatilidad de Internet y con la edad de los alumnos, que han nacido en esta cultura, y con el declive de la prensa escrita en favor de los documentos electrónicos.

La mayoría de los términos identificados $(79,6 \%)$ se han utilizado de forma metafórica, ocupando un lugar importante el término 'crisis', que ya estaba presente en los tratados hipocráticos como manifestación de un cambio súbito del curso de la enfermedad, descrito en los siguientes términos: 'hay crisis en las enfermedades cuando éstas aumentan o se debilitan, se transforman en otras afecciones o llegan a su fin' [19]. Este uso metafórico ha sido resaltado por diferentes autores $[1,2,7,20]$ que, como López-Piñero y Terrada-Ferrandis [21], entienden que 'la prensa no transmite un conocimiento del vocabulario médico real porque utiliza un lenguaje utilitarista, necesario para una comunicación directa entendible (p. ej., dónde asienta una lesión deportiva o la naturaleza de la misma) o recurre a metáforas coloquialistas consagradas por el uso'

En síntesis, nuestro estudio revela que, a pesar de la ventana de observación tan estrecha, existe abundante información de salud en la prensa nacional y regional, como indican tanto el número de artículos como de palabras identificadas por los alumnos, lo que confiere a la prensa un papel informativo y divulgativo de primer orden. Por tanto, consideramos que la iniciativa es útil para los estudiantes del grado en medicina y satisfactoria para el profesorado porque, como indica Bourdieu [6], 'enseñar a cada persona el arte de elaborar su propia retórica es una obra de salud pública'.

\section{Bibliografía}

1. Reboiras JJD, Fidalgo JM, Iglesias MS. Conceptos básicos en terminología médica. Madrid: Alhambra; 1982.

2. Ordóñez-Gallego A. Lenguaje médico. Modismos, tópicos y curiosidades. Madrid: Noesis; 1994.

3. Gutiérrez B. El lenguaje de las ciencias. Madrid: Gredos; 2005.

4. Díaz-Rojo JA. Terminología médica y su discurso social. In García de Toro C, García-Izquierdo I, eds. Experiencias de traducción. Reflexiones desde la práctica traductora. Castellón: Universitat Jaume I; 2005. p. 77-91.

5. Díaz-Rojo JA. Retórica científica en la prensa: el hallazgo paleontológico del cráneo de Orce (1983). In Díaz-Rojo JA, ed. La circulación del saber científico en los siglos XIX y xx. Valencia: Universitat de València; 2011. p. 99-128.

6. Bourdieu P. Cuestiones de sociología. Madrid: Akal; 2008

7. Quintana-Cabanas JM. La terminología médica a partir de sus raíces griegas. Madrid: Dykinson; 1989.

8. Sueiro E. Comunicación y ciencia médica. Investigar con animales para curar a personas. Madrid: CSIC; 2010

9. Hidalgo A, Bordallo J, Sánchez M, Cantabrana B. Protagonismo de los alumnos en el aprendizaje. Una experiencia en el primer curso de medicina. Educ Med 2012; 15: 213-9. 
10. Vallano A, Llop R, Bosch M, Danés I. Noticias sobre medicamentos en los suplementos de salud de la prensa española. Med Clin (Barc) 2005; 124: 754-6.

11. Casino G. Los medicamentos en los medios de comunicación. In Baños JE, Bigorra J, eds. La proyección social del medicamento. Barcelona: Fundación Dr. Antonio Esteve; 2007. p. 67-74.

12. Osca J, Díaz, JA, Mínguez O. Análisis del consumo de información en los artículos españoles de terminología médica. Boletín de la Asociación Andaluza de Bibliotecarios; 1999. URL: http://eprints.rclis.org/handle/10760/5991. [15.02.2012].

13. Elías C. Fundamentos de periodismo científico y divulgación mediática. Madrid: Alianza Editorial; 2008.

14. Fundación Dr. Antonio Esteve. Medicina y medios de comunicación. Barcelona; 1997. URL: http://www.esteve.org/ aw/Home/Secciones_Web/Publicacions/Monograf\%EDas/ $\sim$ qk/copyright_Medicina_y_medios/\#descargas. [20.02.2012]

15. Cámara-Hurtado M, López-Cerezo JA. Percepción del interés y la utilidad del conocimiento científico y tecnológico. Madrid: Fundación Española para la Ciencia y Tecnología (FECYT); 2009. URL: http://www.fecyt.es/fecyt/docs/tmp/ -258613466.pdf. [15.02.2012].
16. Observatorio de la Comunicación Científica y Médica de la Universitat Pompeu Fabra. Proyecto Quiral 10 años, medicina y salud en la prensa diaria. Barcelona: Fundación Vila Casas; 2008. URL: http://www.upf.edu/pcstacademy/_docs/Quiral10. pdf. [20.02.2012]

17. Brockman J, ed. El nuevo humanismo y las fronteras de la ciencia. Barcelona: Kairós; 2007.

18. Martín MJ, Rey J. Cicotec. El papel de los científicos en la comunicación de la ciencia y la tecnología a la sociedad: actitudes, aptitudes e implicación. Madrid: Dirección General de Universidades e Investigación, Consejo Superior de Investigaciones Científicas; 2007.

19. Hermosín MA, ed. Tratados hipocráticos. Madrid: Alianza Editorial; 1996.

20. Núñez-Ramos R, Lorenzo-González G. Tres cerditos. Uso, significado y metáforas. Oviedo: Servicio de Publicaciones de la Universidad de Oviedo; 2004.

21. López-Piñero JM, Terrada-Ferrandis ML. Introducción a la terminología médica. 2 ed. Barcelona: Masson; 2005 